\title{
Geschäftsbericht 2015 der FMH Services Genossenschaft
}

Für die FMH Services Genossenschaft war 2015 ein Jahr, das vor allem geprägt war durch zukunftsorientierte Arbeiten am seit dem 1.1.2016 erneuerten Zusammenarbeitsvertrag mit der FMH. Gleichzeitig haben wir die von der FMH Consulting Services erbrachten Dienstleistungen für Ärzte und Praxen im Markt weiterentwickelt und ausgebaut. Leider wurde das Finanzergebnis auch bei uns von den schwierigen Verhältnissen an den Finanzmärkten beeinflusst. Der Geschäftsbericht 2015 kann auf unserer Website (www.fmhservices.ch) abgerufen werden.

Die Verwaltung der FMH Services Genossenschaft hat per Ende 2015 aufgrund statutarischer Beendigung der Amtszeit ihren langjährigen Präsidenten Dr. med. Max Giger verabschiedet. Wir danken Dr. med. Max Giger für seinen grossen, von Erfolg gekrönten Einsatz zu- gunsten der Entwicklung der FMH Services Genossenschaft und wünschen ihm für die Zukunft alles Gute und beste Gesundheit.

Seit dem 1. Januar 2016 darf ich die FMH Services Genossenschaft als Präsident der Verwaltung leiten. Ebenfalls per Anfang dieses Jahres wurde Dr. Remo Osterwalder, Mitglied des Zentralvorstandes FMH, von den Genossenschafterinnen und Genossenschaftern als Vertreter der FMH zum Mitglied der Verwaltung gewählt.

Ich danke der Ärzteschaft für das uns entgegengebrachte Vertrauen und die in den vergangenen Jahren mit der FMH Services Genossenschaft gepflegten Kontakte. Geschäftspartnern und Mitarbeitenden danke ich für das grosse Engagement, das sie der führenden Dienstleistungsorganisation der in der Schweiz tätigen Ärztinnen und Ärzte entgegengebracht haben.

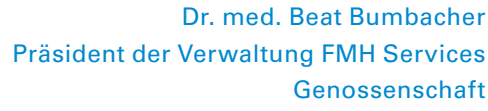

Dr Beat Bumbacher

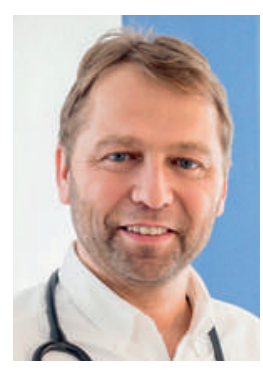

\section{Rapport de gestion 2015 de la coopérative FMH Services}

Pour la coopérative FMH Services, l'année 2015 a notamment été marquée par la révision de la convention de collaboration avec la FMH en vigueur depuis le $1^{\text {er }}$ janvier 2016. En parallèle, nous avons continué à développer et à étendre les prestations de FMH Consulting Services à l'intention des médecins et des cabinets médicaux. Malheureusement, les difficultés sur les marchés financiers ont eu un impact sur nos résultats. Le rapport de gestion 2015 peut être téléchargé sur notre site web (www.fmhservices.ch).

A fin 2015, le conseil d'administration de la coopérative FMH Services a pris congé de son président de longue date, le Dr Max Giger, pour des raisons statutaires. Nous remercions le Dr Max Giger pour son grand engagement couronné de succès en faveur du développe- ment de la coopérative FMH Services et lui adressons tous nos vœux de succès et de santé pour l'avenir. $\mathrm{Au} 1^{\text {er }}$ janvier 2016, j'ai repris la présidence du conseil d'administration. Ce début d'année a également été marqué par la nomination par nos sociétaires du Dr Remo Osterwalder, membre du Comité central de la FMH, au conseil d'administration de FMH Services en tant que délégué de la FMH.

J'en profite pour adresser mes plus vifs remerciements au corps médical pour la confiance témoignée et ses nombreux contacts avec la coopérative FMH Services. Un grand merci également à tous nos partenaires ainsi qu'à toutes les collaboratrices et tous les collaborateurs pour l'engagement dont ils font preuve au sein de la société de services des médecins exerçant en Suisse. 